\title{
Author Index Volume 26 (2016)
}

Aguiar-Furucho, M. A., see Peláez, F. J. R.

Akar, S. A., Kara, S., Latifoğlu, F. \& Bilgiç, V., Analysis of the Complexity Measures in the EEG of Schizophrenia Patients

Alam, M., see Darbin, O.

Alarcón, G., see Spyrou, L.

Alomar, M. L., see Rosselló, J. L.

Andina, D., see Peláez, F. J. R.

Arena, P., Calí, M., Patané, L., Portera, A. \& Strauss, R., A Fly-Inspired Mushroom

Bodies Model for Sensory-Motor Control Through Sequence and Subsequence Learning

Ateş, K., see Aydin, S.

Atienza, M., see Romero-Garcia, R.

Aydin, S., Demirtaş, S., Ateş, K. \& Tunga, M. A., Emotion Recognition with Eigen

Features of Frequency Band Activities Embedded in Induced Brain Oscillations

Mediated by Affective Pictures

Azorín, J. M., see Hortal, E.

Bauer, P. R., see Kalitzin, S. N.

Belardinelli, P., see Knieling, S.

Bilgiç, V., see Akar, S. A.

Bourbakis, N., see Michalopoulos, K.

Calí, M., see Arena, P.

Canals, V., see Rosselló, J. L.

Cantero, J. L., see Romero-Garcia, R.

Carlen, P. L., see Wang, L.

Castellanos-Dominguez, G., see Giraldo-Suarez, E.

Chau, T., see Yin, E.

Chen, L., see Xu, M.

Chuang, C.-H., see Chuang, S.-W.

Chuang, C.-H., see Huang, K.-C.

Chuang, S.-W., Chuang, C.-H., Yu, Y.-H., King, J.-T. \& Lin, C.-T., EEG Alpha and

Gamma Modulators Mediate Motion Sickness-Related Spectral Responses

Crespo-Cano, R., see Martínez-Álvarez, A.

Cuenca-Asensi, S., see Martínez-Álvarez, A.

Daffertshofer, A., see Yang, Y.

D'Angelo, E., see Garrido, J. A.

Darbin, O., Jin, X., Von Wrangel, C., Schwabe, K., Nambu, A., Naritoku, D. K., Krauss,

J. K. \& Alam, M., Neuronal Entropy-Rate Feature of Entopeduncular Nucleus in Rat

Model of Parkinson's Disease

Deiber, M.-P., see Michalopoulos, K.

de la Cal, E., see Villar, J. R.
5 (2016) 1650040

$2(2016) 1650008$

2 (2016) 1550038

4 (2016) 1650016

5 (2016) 1550036

5 (2016) 1650040

6 (2016) 1650035

3 (2016) 1650013

2 (2016) 1650003

3 (2016) 1650013

7 (2016) 1650029

8 (2015) 1650027

5 (2016) 1550042

2 (2016) 1650008

6 (2016) 1650036

6 (2016) 1650035

5 (2016) 1550036

2 (2016) 1650003

8 (2015) 1650044

7 (2016) 1650026

1 (2016) 1650001

3 (2016) 1650010

2 (2016) 1650007

4 (2016) 1650018

2 (2016) 1650007

7 (2016) 1650021

7 (2016) 1650021

1 (2016) 1550031

5 (2016) 1650020

2 (2016) 1550038

6 (2016) 1650036

6 (2016) 1650037 
Demirtaş, S., see Aydin, S.

3 (2016) 1650013

Dewald, J. P. A., see Yang, Y.

Díaz-Tahoces, A., see Martínez-Álvarez, A.

8 (2015) 1650043

7 (2016) 1650021

8 (2015) 1650044

Dufour, S., see Wang, L.

Fernández, E., see Hortal, E.

Fernández, E., see Martínez-Álvarez, A.

Fernandez, E., see Lopez-Gordo, M. A.

Ferrández Vicente, J. M., see Martínez-Álvarez, A.

Garrido, J. A., Luque, N. R., Tolu, S. \& D'Angelo, E., Oscillation-Driven Spike-Timing

Dependent Plasticity Allows Multiple Overlapping Pattern Recognition in Inhibitory

Interneuron Networks

7 (2016) 1650029

7 (2016) 1650021

7 (2016) 1650034

7 (2016) 1650021

Gharabaghi, A., see Knieling, S.

Giraldo-Suarez, E., Martinez-Vargas, J. D. \& Castellanos-Dominguez, G., Reconstruction of Neural Activity from EEG Data Using Dynamic Spatiotemporal Constraints

González, V. M., see Villar, J. R.

Górriz, J. M., see Martinez-Murcia, F. J.

Górriz, J. M., see Ortiz, A.

Grima Murcia, M. D., see Lopez-Gordo, M. A.

Griol, D., Iglesias, J. A., Ledezma, A. \& Sanchis, A., A Two-Stage Combining Classifier

Model for the Development of Adaptive Dialog Systems

He, F., see Xu, M.

Hong, K.-S. \& Naseer, N., Reduction of Delay in Detecting Initial Dips from Functional

Near-Infrared Spectroscopy Signals Using Vector-Based Phase Analysis

Hortal, E., Úbeda, A., Iáñez, E., Azorín, J. M. \& Fernández, E., EEG-Based Detection of

Starting and Stopping During Gait Cycle

$\mathrm{Hu}, \mathrm{D}$. , see Yin, E.

Huang, K.-C., Huang, T.-Y., Chuang, C.-H., King, J.-T., Wang, Y.-K., Lin, C.-T. \&

Jung, T.-P., An EEG-Based Fatigue Detection and Mitigation System

Huang, T.-Y., see Huang, K.-C.

Iáñez, E., see Hortal, E.

Iglesias, J. A., see Griol, D.

Iliya, S. \& Neri, F., Towards Artificial Speech Therapy: A Neural System for Impaired

Speech Segmentation

Innes, C. C. R. H., see Jonmohamadi, Y.

Jin, X., see Darbin, O.

Jones, R. D., see Jonmohamadi, Y.

Jonmohamadi, Y., Poudel, G. R., Innes, C. C. R. H. \& Jones, R. D., Microsleeps are

Associated with Stage-2 Sleep Spindles from Hippocampal-Temporal Network

Jung, T.-P., see Huang, K.-C.

Kalitzin, S. N., Bauer, P. R., Lamberts, R. J., Velis, D. N. \& Thijs, R. D., Automated

Video Detection of Epileptic Convulsion Slowing as a Precursor for Post-Seizure

Neuronal Collapse

Kalitzin, S., see Koppert, M.

Kara, S., see Akar, S. A.

King, J.-T., see Chuang, S.-W.

King, J.-T., see Huang, K.-C.

Knieling, S., Sridharan, K. S., Belardinelli, P., Naros, G., Weiss, D., Mormann, F. \&

Gharabaghi, A., An Unsupervised Online Spike-Sorting Framework

5 (2016) 1650020

5 (2016) 1550042

7 (2016) 1650026

6 (2016) 1650037

7 (2016) 1650024

7 (2016) 1650025

7 (2016) 1650034

1 (2016) 1650002

3 (2016) 1650010

3 (2016) 1650012

7 (2016) 1650029

1 (2016) 1650001

4 (2016) 1650018

4 (2016) 1650018

7 (2016) 1650029

1 (2016) 1650002

6 (2016) 1650023

4 (2016) 1650015

2 (2016) 1550038

4 (2016) 1650015

4 (2016) 1650015

4 (2016) 1650018

8 (2015) 1650027

8 (2015) 1650028

2 (2016) 1650008

2 (2016) 1650007

4 (2016) 1650018

5 (2016) 1550042 
Koppert, M., Kalitzin, S., Velis, D., Lopes Da Silva, F. \& Viergever, M. A., Preventive and Abortive Strategies for Stimulation Based Control of Epilepsy: A Computational Model Study

Krauss, J. K., see Darbin, O.

Lamberts, R. J., see Kalitzin, S. N.

Latifoğlu, F., see Akar, S. A.

Ledezma, A., see Griol, D.

$\mathrm{Li}, \mathrm{C}$., see Li, J.

Li, J., Zhou, W., Yuan, S., Zhang, Y., Li, C. \& Wu, Q., An Improved Sparse

Representation over Learned Dictionary Method for Seizure Detection

Li, M., Li, W. \& Zhou, H., Increasing N200 Potentials Via Visual Stimulus Depicting Humanoid Robot Behavior

Li, W., see Li, M.

Lin, C.-T., see Chuang, S.-W.

Lin, C.-T., see Huang, K.-C.

Liu, J., see Xu, M.

Looney, D., see Tonoyan, Y.

Lopes Da Silva, F., see Koppert, M.

Lopez-Gordo, M. A., Grima Murcia, M. D., Padilla, P., Pelayo, F. \& Fernandez, E., Asynchronous Detection of Trials Onset from Raw EEG Signals

López-Rubio, E., see Palomo, E. J.

Luque, N. R., see Garrido, J. A.

Mandic, D. P., see Tonoyan, Y.

Martínez-Cañada, P., Morillas, C., Pino, B., Ros, E. \& Pelayo, F., A Computational

Framework for Realistic Retina Modeling

Martínez-Álvarez, A., Crespo-Cano, R., Díaz-Tahoces, A., Cuenca-Asensi, S., Ferrández

Vicente, J. M. \& Fernández, E., Automatic Tuning of a Retina Model for a Cortical

Visual Neuroprosthesis Using a Multi-Objective Optimization Genetic Algorithm

Martinez-Murcia, F. J., Górriz, J. M., Ramírez, J. \& Ortiz, A., A Structural

Parametrization of the Brain Using Hidden Markov Models-Based Paths in Alzheimer's Disease

Martinez-Vargas, J. D., see Giraldo-Suarez, E.

Martín-Lopez, D., see Spyrou, L.

Menéndez, E., see Villar, J. R.

Michalopoulos, K., Zervakis, M., Deiber, M.-P. \& Bourbakis, N., Classification of EEG

Single Trial Microstates Using Local Global Graphs and Discrete Hidden Markov

Models

Milanowski, P. \& Suffczynski, P., Seizures Start without Common Signatures of Critical

Transition

Ming, D., see $\mathrm{Xu}, \mathrm{M}$.

Morillas, C., see Martínez-Cañada, P.

Mormann, F., see Knieling, S.

Morro, A., see Rosselló, J. L.

Munilla, J., see Ortiz, A.

Nambu, A., see Darbin, O.

Naritoku, D. K., see Darbin, O.

Naros, G., see Knieling, S.

Naseer, N., see Hong, K.-S.

Neri, F., see Iliya, S.
8 (2015) 1650028

2 (2016) 1550038

8 (2015) 1650027

2 (2016) 1650008

1 (2016) 1650002

1 (2016) 1550035

1 (2016) 1550035

1 (2016) 1550039

1 (2016) 1550039

2 (2016) 1650007

4 (2016) 1650018

3 (2016) 1650010

2 (2016) 1650005

8 (2015) 1650028

7 (2016) 1650034

4 (2016) 1650019

5 (2016) 1650020

2 (2016) 1650005

7 (2016) 1650030

7 (2016) 1650021

7 (2016) 1650024

7 (2016) 1650026

4 (2016) 1650016

6 (2016) 1650037

6 (2016) 1650036

8 (2015) 1650053

3 (2016) 1650010

7 (2016) 1650030

5 (2016) 1550042

5 (2016) 1550036

7 (2016) 1650025

2 (2016) 1550038

2 (2016) 1550038

5 (2016) 1550042

3 (2016) 1650012

6 (2016) 1650023 
Nishimura, H., see Nobukawa, S.

5 (2016) 1550040

Nobukawa, S. \& Nishimura, H., Enhancement of Spike-Timing-Dependent Plasticity in

Spiking Neural Systems with Noise

5 (2016) 1550040

O'Hara, B. F., see Yaghouby, F.

Oliver, A., see Rosselló, J. L.

Ortiz, A., Munilla, J., Górriz, J. M. \& Ramírez, J., Ensembles of Deep Learning

Architectures for the Early Diagnosis of the Alzheimer's Disease

Ortiz, A., see Martinez-Murcia, F. J.

Padilla, P., see Lopez-Gordo, M. A.

Palomo, E. J. \& López-Rubio, E., Learning Topologies with the Growing Neural Forest

Patané, L., see Arena, P.

Peláez, F. J. R., Aguiar-Furucho, M. A. \& Andina, D., Intrinsic Plasticity for Natural

Competition in Koniocortex-Like Neural Networks

Pelayo, F., see Lopez-Gordo, M. A.

Pelayo, F., see Martínez-Cañada, P.

Peng, H., Wang, J., Shi, P., Pérez-Jiménez, M. J. \& Riscos-Núñez, A., An Extended

Membrane System with Active Membranes to Solve Automatic Fuzzy Clustering

Problems

Pérez-Jiménez, M. J., see Peng, H.

Pino, B., see Martínez-Cañada, P.

Portera, A., see Arena, P.

Poudel, G. R., see Jonmohamadi, Y.

Qi, H., see Xu, M.

Ramírez, J., see Martinez-Murcia, F. J.

Ramírez, J., see Ortiz, A.

Riscos-Núnez, A., see Peng, H.

Romero-Garcia, R., Atienza, M. \& Cantero, J. L., Different Scales of Cortical

Organization are Selectively Targeted in the Progression to Alzheimer's Disease

Ros, E., see Martínez-Cañada, P.

Rosselló, J. L., Alomar, M. L., Morro, A., Oliver, A. \& Canals, V., High-Density

Liquid-State Machine Circuitry for Time-Series Forecasting

Saab, R., see Yin, E.

Sanchis, A., see Griol, D.

Sanei, S., see Spyrou, L.

Schouten, A. C., see Yang, Y.

Schouten, A. C., see Yang, Y.

Schwabe, K., see Darbin, O.

Sedano, J., see Villar, J. R.

Shi, P., see Peng, H.

Shull, P. B., see Zhang, D.

Solis-Escalante, T., see Yang, Y.

Solis-Escalante, T., see Yang, Y.

Spyrou, L., Martín-Lopez, D., Valentín, A., Alarcón, G. \& Sanei, S., Detection of

Intracranial Signatures of Interictal Epileptiform Discharges from Concurrent Scalp

EEG

4 (2016) 1650017

5 (2016) 1550036

7 (2016) 1650025

7 (2016) 1650024

7 (2016) 1650034

4 (2016) 1650019

6 (2016) 1650035

5 (2016) 1650040

7 (2016) 1650034

7 (2016) 1650030

3 (2016) 1650004

3 (2016) 1650004

7 (2016) 1650030

6 (2016) 1650035

4 (2016) 1650015

3 (2016) 1650010

7 (2016) 1650024

7 (2016) 1650025

3 (2016) 1650004

2 (2016) 1650003

7 (2016) 1650030

5 (2016) 1550036

1 (2016) 1650001

1 (2016) 1650002

4 (2016) 1650016

1 (2016) 1550031

8 (2015) 1650043

2 (2016) 1550038

6 (2016) 1650037

3 (2016) 1650004

2 (2016) 1650006

1 (2016) 1550031

8 (2015) 1650043

Sridharan, K. S., see Knieling, S.

Strauss, R., see Arena, P.

Suffczynski, P., see Milanowski, P.

Sunderam, S., see Yaghouby, F.

4 (2016) 1650016

5 (2016) 1550042

6 (2016) 1650035

8 (2015) 1650053

4 (2016) 1650017 
Thijs, R. D., see Kalitzin, S. N.

8 (2015) 1650027

Tolu, S., see Garrido, J. A.

Tonoyan, Y., Looney, D., Mandic, D. P. \& Van Hulle, M. M., Discriminating Multiple

Emotional States from EEG Using a Data-Adaptive, Multiscale Information-Theoretic Approach

Tunga, M. A., see Aydin, S.

Úbeda, A., see Hortal, E.

Valentín, A., see Spyrou, L.

Valiante, T. A., see Wang, L.

van der Helm, F. C. T., see Yang, Y.

van der Helm, F. C. T., see Yang, Y.

Van Hulle, M. M., see Tonoyan, Y.

Van Hulle, M. M., see Wittevrongel, B.

Velis, D. N., see Kalitzin, S. N.

Velis, D., see Koppert, M.

Vergara, P., see Villar, J. R.

Viergever, M. A., see Koppert, M.

Villar, J. R., Vergara, P., Menéndez, E., de la Cal, E., González, V. M. \& Sedano, J.,

Generalized Models for the Classification of Abnormal Movements in Daily Life and its

Applicability to Epilepsy Convulsion Recognition

Von Wrangel, C., see Darbin, O.

Wan, B., see Xu, M.

Wang, J., see Peng, H.

Wang, L., Dufour, S., Valiante, T. A. \& Carlen, P. L., Extracellular Potassium and

Seizures: Excitation, Inhibition and the Role of Ih

Wang, Y.-K., see Huang, K.-C.

Weiss, D., see Knieling, S.

Wittevrongel, B. \& Van Hulle, M. M., Faster P300 Classifier Training Using

Spatiotemporal Beamforming

$\mathrm{Wu}, \mathrm{Q}$, see $\mathrm{Li}, \mathrm{J}$.

$\mathrm{Wu}, \mathrm{Q}$, see $\mathrm{Xu}, \mathrm{F}$.

$\mathrm{Wu}$, Q., see Yuan, S.

Xu, F., Zhou, W., Zhen, Y., Yuan, Q. \& Wu, Q., Using Fractal and Local Binary Pattern Features for Classification of ECOG Motor Imagery Tasks Obtained from the Right

Brain Hemisphere

$\mathrm{Xu}, \mathrm{F}$., see Zhang, D.

$\mathrm{Xu}, \mathrm{H}$., see Zhang, D.

Xu, M., Liu, J., Chen, L., Qi, H., He, F., Zhou, P., Wan, B. \& Ming, D., Incorporation of Inter-Subject Information to Improve the Accuracy of Subject-Specific P300 Classifiers

Yaghouby, F., O'Hara, B. F. \& Sunderam, S., Unsupervised Estimation of Mouse Sleep

Scores and Dynamics Using a Graphical Model of Electrophysiological Measurements

Yang, Y., Solis-Escalante, T., Yao, J., Daffertshofer, A., Schouten, A. C. \& van der Helm,

F. C. T., A General Approach for Quantifying Nonlinear Connectivity in the Nervous System Based on Phase Coupling

Yang, Y., Solis-Escalante, T., Yao, J., van der Helm, F. C. T., Dewald, J. P. A. \&

Schouten, A. C., Nonlinear Connectivity in the Human Stretch Reflex Assessed by

Cross-Frequency Phase Coupling

5 (2016) 1650020

$2(2016) 1650005$

3 (2016) 1650013

7 (2016) 1650029

4 (2016) 1650016

8 (2015) 1650044

1 (2016) 1550031

8 (2015) 1650043

2 (2016) 1650005

3 (2016) 1650014

8 (2015) 1650027

8 (2015) 1650028

6 (2016) 1650037

8 (2015) 1650028

6 (2016) 1650037

2 (2016) 1550038

3 (2016) 1650010

3 (2016) 1650004

8 (2015) 1650044

4 (2016) 1650018

5 (2016) 1550042

3 (2016) 1650014

1 (2016) 1550035

6 (2016) 1650022

3 (2016) 1650011

6 (2016) 1650022

2 (2016) 1650006

2 (2016) 1650006

3 (2016) 1650010

4 (2016) 1650017

Yao, J., see Yang, Y.

Yao, J., see Yang, Y.

8 (2015) 1650043

1 (2016) 1550031

8 (2015) 1650043 
Yin, E., Zeyl, T., Saab, R., Hu, D., Zhou, Z. \& Chau, T. An Auditory-Tactile Visual Saccade-Independent P300 Brain-Computer Interface

1 (2016) 1650001

Yu, Y.-H., see Chuang, S.-W.

2 (2016) 1650007

Yuan, Q., see Xu, F.

6 (2016) 1650022

Yuan, S., Zhou, W., Wu, Q. \& Zhang, Y., Epileptic Seizure Detection with Log-Euclidean Gaussian Kernel-Based Sparse Representation

3 (2016) 1650011

Yuan, S., see Li, J.

Zervakis, M., see Michalopoulos, K.

Zeyl, T., see Yin, E.

Zhang, D., Xu, F., Xu, H., Shull, P. B. \& Zhu, X., Quantifying Different Tactile

Sensations Evoked by Cutaneous Electrical Stimulation Using Electroencephalography

\section{Features}

Zhang, Y., see Li, J.

Zhang, Y., see Yuan, S.

Zhen, Y., see Xu, F.

Zhou, H., see Li, M.

Zhou, P., see Xu, M.

1 (2016) 1550035

6 (2016) 1650036

1 (2016) 1650001

Zhou, W., see Li, J.

Zhou, W., see Xu, F.

Zhou, W., see Yuan, S.

(2016) 1650006

1 (2016) 1550035

3 (2016) 1650011

6 (2016) 1650022

1 (2016) 1550039

3 (2016) 1650010

1 (2016) 1550035

6 (2016) 1650022

3 (2016) 1650011

Zhou, Z., see Yin, E.

1 (2016) 1650001

Zhu, X., see Zhang, D.

2 (2016) 1650006 